\title{
EFECTOS DE LA LOMBRIZ ROJA CALIFORNIANA (Eisenia foetida), SOBRE EL CRECIMIENTO DE MICROORGANISMOS EN SUELOS CONTAMINADOS CON MERCURIO DE SEGOVIA, ANTIOQUIA
}

\author{
EFFECTS OF EARTHWORMS (Eisenia foetida) ON THE MICROORGANIS \\ GROWTH IN POLLUTION SOILS WITH MERCURY OF SEGOVIA, ANTIOQUIA
}

Isabel C. Zapata', Laura Martínez², Estefanía Posada³, María E. González ${ }^{4}$, Juan F. Saldarriaga ${ }^{5}$

Fecha de recepción: 11 de junio de 2016

Fecha de revisión: 8 de septiembre de 2016

Fecha de aprobación: 23 de octubre de 2016

Referencia: I. C. Zapata, L. Martínez, E. Posada, M. E. González, J. F. Saldarriaga (2017). Efectos de la lombriz roja californiana (Eisenia Foetida), sobre el crecimiento de microorganismos en suelos contaminados con mercurio de Segovia, Antioquia. Ciencia e Ingeniería Neogranadina, 27 (1), pp. 77-90, D0l: http://dx.doi.org/10.18359/rcin.1911

\section{RESUMEN}

Dentro de las actividades que alteran la composición del suelo está la minería, que causa el aumento de daños visibles al suelo mediante la creación de minas a cielo abierto, escombreras, estanques de residuos y otras instalaciones de minas. El objetivo principal de este trabajo fue evaluar el efecto de la lombriz roja californiana sobre el crecimiento de microorganismos en suelos contaminados con mercurio en el municipio de Segovia, Antioquia. Estos suelos representan una problemática importante debido a la acumulación de mercurio en exceso que

1. Ingeniera Ambiental, Colegio Mayor de Antioquia, Medellín - Colombia, icristinazapata@est.colmayor.edu.co

2. Ingeniera Ambiental, Colegio Mayor de Antioquia, Medellín - Colombia, Imartineza@est.colmayor.edu.co

3. Ingeniera Ambiental, Colegio Mayor de Antioquia, Medellín - Colombia, eposadam@est.colmayor.edu.co

4. Especialista es gestión ambiental. Bacterióloga y Bioanalista, Grupo de investigación BIOCIENCIAS, Profesora Tiempo Completo, Colegio Mayor de Antioquia, Medellín - Colombia, maria1.gonzalez@colmayor.edu.co

5. Doctor en Ingeniería de Procesos Químicos y Desarrollo Sostenible. Grupo de investigaciones GEMA, Profesor Tiempo Completo, Universidad de Medellín, Medellín - Colombia, jsaldarriaga@udem.edu.co 
ocasiona la pérdida de la calidad del suelo. Para este estudio se tomaron cuatro tratamientos en diferentes concentraciones de mercurio y un tratamiento más como blanco con lombrices rojas californianas (Eisenia foetida). Para esto, se evaluó el crecimiento de microorganismos a través de diferentes cultivos en el laboratorio, encontrando un crecimiento satisfactorio de los mismos luego de 25 y 90 días de tratamiento. De acuerdo al comportamiento observado, se evidencia una inmovilización del mercurio y una adaptación de los microorganismos al suelo contaminado, mostrando un aumento significativo de estos con el transcurso del tiempo.

Palabras clave: biorremediación, microorganismos, Eisenia foetida, suelo, contaminación por mercurio.

\section{ABSTRACT}

Mining is among the activities that alter soil composition the most, which causes visible ground damage by open-pit mines, mine waste tips, tailings ponds and other mine facilities. The main goal of this work was to assess the effect of earthworm on the microorganism growth in mercury polluted soil exposed during the mining activities in the Segovia municipality. These soils represent a significant problem due to the mercury accumulations that causes the loss of soil quality and fertility. For the evaluation have been used contaminated soil from Segovia, which has been mixed with fertile soil in different proportions (30-70, 50-50 and 70-30 contaminated soil - fertile soil), also have been used two controls, fertile soil with earthworm (Eisenia foetida) and soil contaminated without earthworm. Different laboratory cultures were used to study microorganism growth, finding satisfactory growth of microorganisms after 25 and 90 days of treatment. According to the observed behavior, immobilization of mercury and microorganisms adaptation to the environment was evident and grew with time.

Keywords: Bio-remediation, microorganisms, Eisenia foetida, soil, mercury polluted

\section{INTRODUCCIÓN}

En recientes años la atención pública se ha enfocado en la contaminación ambiental y sus efectos en las personas y otras especies. La mayor cantidad de contaminantes son residuos que provienen de la sociedad industrializada y urbanizada junto con la gran cantidad de químicos, nuevos y viejos, necesarios para mantener una sociedad rica. El suelo es el receptor primario o secundario, de muchos de estos residuos. Luego de que estos materiales entran al suelo, comienzan a hacer parte de un ciclo que afecta a todas las formas de vida [1]. Dentro de las actividades que alteran la composición del suelo está la minería, que causa el aumento de daños visibles al suelo mediante la creación de minas a cielo abierto, escombreras, estanques de residuos y otras instalaciones de minas. El esfuerzo principal de las investigaciones está dirigido a desarrollar métodos apropiados de gestión de dichos 
sitios después de su abandono para neutralizar los efectos potencialmente adversos sobre el ambiente local [2].

Miles de hectáreas en toda la tierra reciben gran variedad de contaminantes [3]. De estos, los metales pesados son contaminantes irreversibles del suelo y uno de los problemas más nocivos de las últimas décadas [4]. La liberación anual de metales pesados alcanza las 22.000 toneladas métricas para Cd, 939.000 para Cu, 1.350 .000 para Zn y 738.000 para $\mathrm{Pb}$; durante la pasada década $[5,6]$ reportaron que para finales del 2004 la degradación del suelo asociado con actividades de minería, había alcanzado 3.2 millones de hectáreas (ha).

El mercurio $(\mathrm{Hg})$, la contaminación ambiental está creciendo a nivel mundial $[7,8]$. El Hg puede estar presente de forma natural en los suelos o como el resultado de la actividad humana, como la minería de oro $[9,10]$. A pequeña escala o artesanal la minería de oro con frecuencia usa tecnologías rudimentarias que involucran la amalgamación con $\mathrm{Hg}$ en los procesos de extracción. Aunque en el mundo existen otras técnicas de explotación, este método de extracción el cual data del tiempo de Nero (54-68 A.C), es ampliamente utilizado porque requiere de una pequeña inversión inicial y mínimo conocimiento de la tecnología $[8,9]$. El proceso de amalgamación es ineficiente para la extracción del oro e inevitablemente introduce $\mathrm{Hg}$ al ambiente (aire, agua y suelo). Según [9], a 20131.000 ton de $\mathrm{Hg}$ han sido derramadas al ambiente en procesos de amalgamación.

El mercurio ingresa en los residuos dejados en el proceso de minería y es dominado principalmente por la especie inorgánica de $\mathrm{Hg}$ (II). El mercurio puede ser metilado como $\mathrm{MeHg}$, siendo este la forma más tóxica [11-
14]. El Hg (II) y el MeHg en el suelo pueden bioacumularse en los organismos acuáticos y ser transferido a través de la cadena alimenticia en el tiempo, resultando ser una amenaza para los seres humanos y la vida silvestre $[12,15]$. Las principales fuentes de emisión de mercurio al ambiente de forma natural son las erupciones volcánicas, la erosión de suelos, la liberación desde las rocas que contienen mercurio así como la descomposición bacterial de compuestos orgánicos con contenidos de mercurio $[16,17]$.

El mercurio es conocido por sus propiedades tóxicas [18]. Los efectos a la salud del Hg dependen de la dosis y duración de la exposición. La exposición de la población al mercurio trae efectos adversos como problemas renales, cardiovasculares, efectos en el sistema reproductivo e inmunológico $[6,19,20]$. El uso de mercurio a pequeña escala, industrial y artesanal para la extracción de oro resulta en la volatilización de un estimado de 300 t de $\mathrm{Hg}$ directamente a la atmósfera anualmente, y de 700 t que son descargadas vía residuos de minería al aire, suelo, ríos y lagos $[8,20]$ y que tiene como resultado miles de sitios contaminados [9]. Las grandes concentraciones en el suelo provocan un ambiente tóxico para los macro y microorganismos, llevando a la infertilidad, baja calidad y salud del suelo. Otro factor importante del mercurio son sus características bioacumuladoras en los tejidos, que puede incorporase en la cadena trófica de los seres vivos causando afectaciones a la salud humana [21-23].

La biorremediación de suelos es un tema que se ha venido investigando en los últimos años debido a sus ventajas frente a la remediación físico-química, en las que se incluyen los bajos costos y los impactos positivos socioambientales [24]. La remediación de suelos 
contaminados usando lombriz de tierra y plantas parece ser una tecnología rentable y ambientalmente amigable. La lombriz de tierra mejora la estructura del suelo, contribuye a la descomposición de la materia orgánica y mejora el ciclo de nutrientes, además juega un papel clave en la evaluación del riesgo eco-toxicológico terrestre [2527]. La implementación de lombrices para la biorremediación es una técnica novedosa que ha mostrado que las capacidades de asimilación de metales por vía digestiva es prácticamente independiente del $\mathrm{pH}$ del suelo [27]. A través de las secreciones mucilaginosas y la transformación de materia orgánica, las lombrices incrementan la actividad microbial y la disponibilidad de los nutrientes, de igual manera se ha reportado que las lombrices pueden estimular las bacterias, hongos y especies relacionadas con la degradación del pentaclorofenol [28-32].

La lombriz tiene la capacidad de asimilar elevadas concentraciones de algunos compuestos protóxidos y metales (Cu, Fe, Cd, Pb, $\mathrm{Zn)}$, sin observarse síntomas aparentes de intoxicación a corto plazo [27].

En este estudio se evalúa la eficacia de las lombrices rojas californianas para movilizar y degradar el mercurio presente en los suelos mineros de Segovia, Antioquia.

\section{MATERIALES Y MÉTODOS}

Para este trabajo se tomó suelo contaminado del municipio de Segovia, Colombia, el cual fue mezclado en suelo fértil comprado en un vivero. Las concentraciones fueron de 70-30, 30-70 y 50-50 de suelo contaminado con respecto a suelo fértil. Para el proceso de biorremediación se utilizó lombriz roja californiana (Eisenia foetida). Para el aislamiento de los microorganismos se emplearon diferentes agares con el fin de identificar levaduras y hongos (papa dextrosa agar-PDA- (Merck) agar plate count (Merck)), para el aislamiento de mesófilos se usó agar plate count (Merck), para enterococos se utilizó agar enterococoscell (BBL) y para baterías se dispusieron dos agares EMB (Merck) y azida+sangre (Dibico) y para el aislamiento de pseudomonas se destinó agar cetrimide (BBL). Todos los conteos se realizaron por medio de recuento y diferencial en placa por siembra en superficie [33]. Las siembra de microorganismos se llevó a cabo mediante diluciones seriadas para los cuatros sistemas evaluados [34].

Para el montaje se utilizaron tres concentraciones diferentes y una muestra control (Tabla 1), el tiempo de ensayo fue de 90 días, dividido en tres etapas de muestreo y análisis en el laboratorio: al primer día, a los 15 días y a los 90 días con el que se finaliza el proceso de remediación. Los primeros 15 días se les realizó seguimiento diario a los sistemas y luego cada 8 días.

Tabla 1. Concentración de suelo y lombrices.

\begin{tabular}{|c|c|c|c|c|c|}
\hline \multicolumn{5}{|c|}{ Tratamientos } \\
\hline Suelo & T1 & T2 & T3 & T4 & T5 \\
\hline Contaminado & $100 \%$ & $70 \%$ & $50 \%$ & $30 \%$ & \\
\hline Fértil & & $30 \%$ & $50 \%$ & $70 \%$ & $100 \%$ \\
\hline
\end{tabular}

Fuente: Elaboración propia.

Se observa en la Tabla 1 que se realizaron diferentes mezclas con el fin de evaluar la efectividad del proceso de biorremediación partiendo de un suelo sin contaminar que evalúa el comportamiento de las lombrices en un ambiente 
inocuo y se le aumenta la concentración de contaminante hasta llegar a una concentración extrema de $70 \%$. Esta mezcla es necesaria ya que cuando se realiza el tratamiento de biorremediación se debe partir de un suelo fértil para darle las condiciones apropiadas a la lombriz como organismo vivo [35].

Para el análisis de datos se hizo por medio de una ANOVA, mediante el software estadístico STATGRAPHIC.

\section{RESULTADOS Y DISCUSIÓN}

Durante la evaluación del efecto de las lombrices rojas sobre el crecimiento de microorganismos en suelo procedente del municipio de Segovia, Antioquia, con un suelo altamente contaminado con mercurio originario de los procesos de extracción del oro, se observa cómo la colonia de microorganismos es bastante baja, encontrando solo levaduras, mesófilos y enterobacterias en el primer día (entre $10^{3}$ y $10^{4} \mathrm{UFC} / \mathrm{g}$ ), evidenciando los problemas de contaminación del suelo, debido a que su rango de microorganismos es bastante bajo con respecto a lo recomendado por la literatura para un suelo sin contaminación (entre $10^{7}$ y $10^{9}$ ) UFC/g.

En la Figura 1 se observa cómo los microorganismos aumentaron luego de 90 días de tratamiento en todos los sistemas analizados a excepción del tratamiento 1 en el cual disminuyeron notablemente. De igual manera, se muestra que todos los sistemas cuando comenzaron su proceso de biorremediación más o menos en $10^{3} \mathrm{UFC} / g$, aumentan después de los 90 días, esto se debe a la interacción que se desarrolla en el sistema entre lombrices y microorganismos. Durante el proceso se encontraron similares comportamientos de la

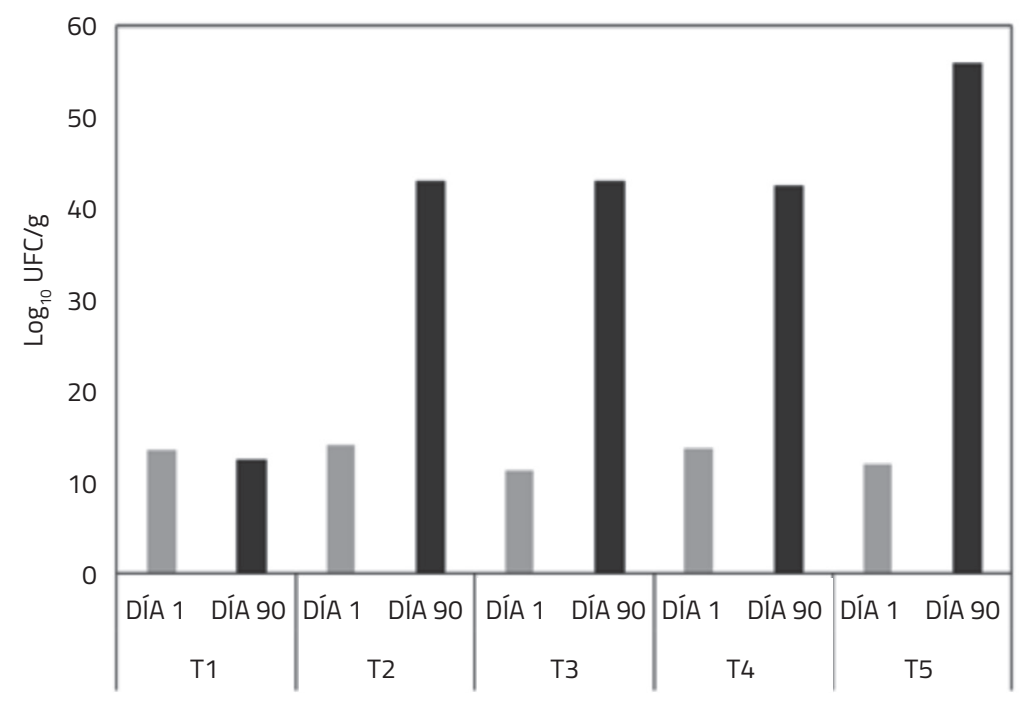

Figura 1. Número total de microorganismos.

Fuente: Elaboración propia. 
lombriz roja con respecto a los encontrados en el trabajo realizado por [35], en el cual el número de individuos disminuyó durante las primeras semanas, mientras se adaptaban al proceso pero luego de 30 días alcanzaron niveles de reproducción entre ellas, de igual manera los microorganismos del suelo aumentan ya que las relaciones existentes en el suelo y los exudados de las lombrices favorecen el aumento de la comunidad bacteriana en el suelo tratado [36].

En la Figura 2 y la Tabla 2 se muestra cómo las enterobacterias, los microorganismos mesófilos y las levaduras son los de mayor presencia durante todo el tiempo de tratamiento, encontrando que al día 15 presentan un aumento considerable, pero para el día 90 disminuyen, la cual se puede atribuir al consumo de nutrientes, quedando poco disponibles en los sistemas tratados, este comportamiento es similar al descrito por [37], debido a que las lombrices mejoran la cantidad de materia orgánica debido a sus deyecciones aumentando nutrientes esenciales como N, P y $\mathrm{K}$, también el $\mathrm{N}$ y $\mathrm{P}$ orgánicos se transforman fácilmente en formas más asimilables [38]. También, [38] argumentan que si el lombricompuesto se incorpora a suelos deficientes en bacterias como Azobacter sp. y
Azospirillium sp., este ayuda a mejorar la fijación del nitrógeno atmosférico.

De igual manera, se evidencia que las enterobacterias (Figura 2A) al inicio del proceso son menores con respecto a los mesófilos y levaduras (Figura 2B y Figura 2C), pero para el día 15 su número de colonias aumenta considerablemente e incluso en el día 90, mientras que para las levaduras (Figura 2C) se da una disminución del número de colonias en todos los tratamientos para el día 90.

En la Figura 3A se muestra un comportamiento similar para las levaduras en el día 15 para los tratamientos T2 y T3, de igual manera se observa que tanto las levaduras, como los mesófilos y enterobacterias para el tratamiento cinco disminuyen notablemente, esto se puede atribuir a la contaminación del suelo por el mercurio, principalmente. Así mismo, se observa que en todos los tratamientos con lombrices los microorganismos fueron incrementando con el tiempo.

En la Figura 3B a diferencia de la Figura 3A, se observa que hay menos cantidad de microorganismos y es en el día 90 donde aumentan, esto puede ser atribuible a que el suelo ha sufrido

Tabla 2. Cantidad de microrganismos por tratamiento y día de muestreo (UFC/g)

\begin{tabular}{|c|c|c|c|}
\hline TRATAMIENTO & DIA 1 & DIA 15 & DIA 90 \\
\hline T1 & $1.06 \mathrm{E}+05$ & $1.07 \mathrm{E}+11$ & $5.40 \mathrm{E}+03$ \\
\hline $\mathrm{T} 2$ & $1.65 \mathrm{E}+05$ & $6.01 \mathrm{E}+10$ & $1.68 \mathrm{E}+10$ \\
\hline $\mathrm{T3}$ & $2.80 \mathrm{E}+04$ & $5.72 \mathrm{E}+10$ & $9.34 \mathrm{E}+10$ \\
\hline $\mathrm{T} 4$ & $1.10 \mathrm{E}+05$ & $8.26 \mathrm{E}+10$ & $8.76 \mathrm{E}+10$ \\
\hline $\mathrm{T} 5$ & $6.10 \mathrm{E}+04$ & $5.54 \mathrm{E}+10$ & $1.75 \mathrm{E}+11$ \\
\hline
\end{tabular}

Fuente: Elaboración propia. 

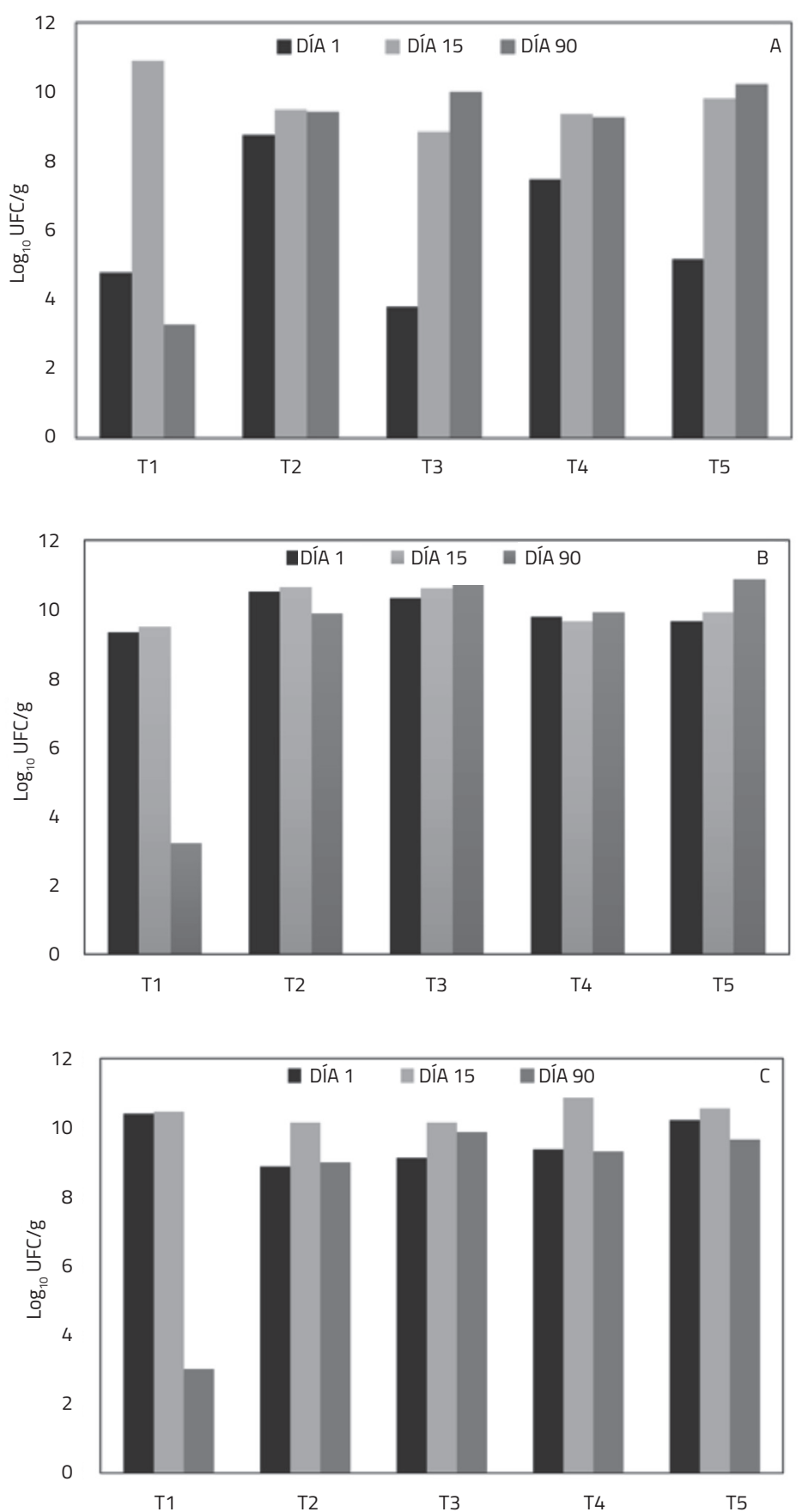

Figura 2. Microorganismos durante el proceso de biorremediación en Log10 UFC/g. (A. Enterobacterias, B. Mesófilos, C. Levaduras).

Fuente: Elaboración propia. 

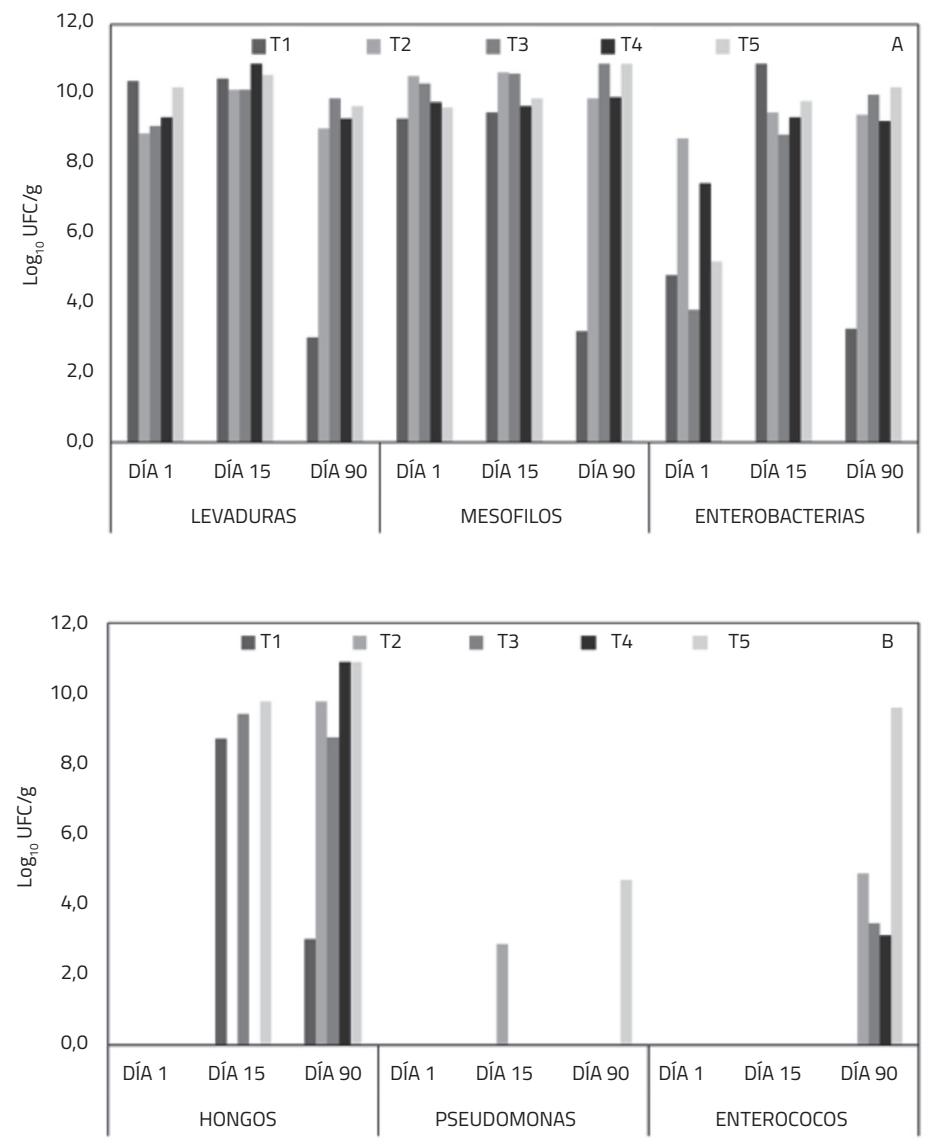

Figura 3. Microorganismos durante el proceso de biorremediación en Log10 UFC/g. (A. Levaduras, mesófilos, enterobacterias. B. Hongos, pseudomonas, enterococos).

Fuente: Elaboración propia.

cambios considerables y que ha permitido el crecimiento de microorganismos, mejorando las condiciones iniciales del suelo tratado. De igual manera, se observa cómo los enterococos aparecen solamente en el día 90 para los sistemas tratados, así mismo se ve cómo los suelos tratados a medida que pasa el tiempo, se van comportando similar al suelo fértil.

De acuerdo al comportamiento observado, se evidencia un aumento en el crecimiento de microorganismos en suelo tratado, estos efectos se deben a la simbiosis que se produce entre las lombrices y los microorganismos del medio, tanto los que estaban en el suelo cuando se inició el proceso como las nuevas colonias registradas, evidenciándose una interacción lombrices-microorganismos [18,39].

En la Tabla 3, se muestra el análisis de varianza para todos los tratamientos, en el cual se puede observar cómo con el transcurso del tiempo estos aumentan y se vuelven fundamentales en el crecimiento de los microorganismos. 
Tabla 3. Análisis de varianza para todos los tratamientos y días evaluados.

\begin{tabular}{|c|c|c|c|c|c|}
\hline Fuente & $\begin{array}{c}\text { Suma de } \\
\text { cuadrados }\end{array}$ & $\begin{array}{c}\text { Grados de } \\
\text { Libertad }\end{array}$ & $\begin{array}{c}\text { Cuadrado } \\
\text { Medio }\end{array}$ & $\begin{array}{c}\text { Componente } \\
\text { de la Varianza }\end{array}$ & Valor-P \\
\hline EFECTOS PRINCIPALES & & & & & \\
\hline A: Días & $1.48454 \times 10^{21}$ & 2 & $7.42268 \times 10^{20}$ & 2.70 & 0.0796 \\
\hline B: Microorganismos & $5.53949 \times 10^{21}$ & 5 & $1.1079 \times 10^{21}$ & 4.03 & 0.0047 \\
\hline C: Tratamientos & $6.42755 \times 10^{20}$ & 4 & $1.60689 \times 10^{20}$ & 0.58 & 0.6761 \\
\hline INTERACCIONES & & & & & 0.0364 \\
\hline AB & $6.1073 \times 10^{21}$ & 10 & $6.1073 \times 10^{20}$ & 2.22 & 0.2395 \\
\hline AC & $3.01392 \times 10^{21}$ & 8 & $3.7674 \times 10^{20}$ & 1.37 & 0.1738 \\
\hline BC & $7.76504 \times 10^{21}$ & 20 & $3.88252 \times 10^{20}$ & 1.41 & \\
\hline RESIDUAL & $1.10076 \times 10^{22}$ & 40 & $2.7519 \times 10^{20}$ & & \\
\hline TOTAL (CORREGIDO) & $3.55606 \times 10^{22}$ & 89 & & & \\
\hline
\end{tabular}

Fuente: Elaboración propia.

Por otro lado, se realizó una prueba de rangos múltiples en la cual se determinó que durante el tiempo de remediación aplicado al suelo contaminado con mercurio los microorganismos al día 25 han conseguido una adaptación al medio y que al día 90 las unidades formadoras no tienen un cambio estadístico significativo con respecto al día 25 (Figura 4). De igual manera, se observa que los microorganismos de mayor presencia en todos los sistemas son los mesófilos segui-

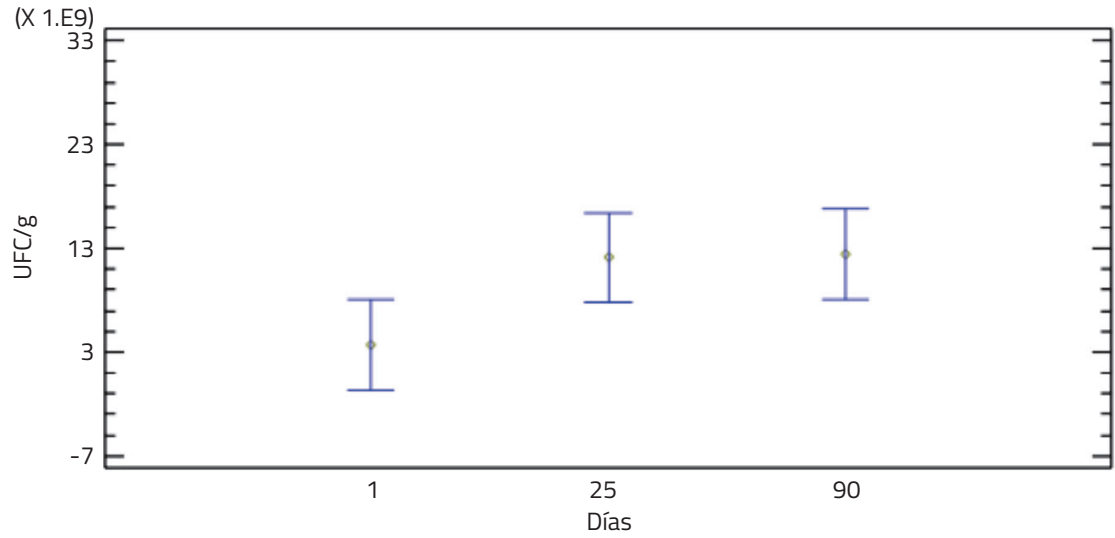

Figura 4. Gráfico de medias para las UFC por días de tratamiento.

Fuente: Elaboración propia. 
dos por las levaduras y los hongos (Figura 5), resultados que son muy similares a los hallados por $[18,40,41]$, que encontraron que hongos de Aspergillus flavus tienen una capacidad de tolerar contaminación por mercurio (II) con una concentración superior a $100 \mathrm{mg} / \mathrm{l}$.

También en la Figura 6, se muestra cómo el comportamiento del crecimiento de los microorganismos evaluado como unidades formadoras de colonias que en todos los tratamientos es similar y que en ninguno hubo una diferencia significativa, evidenciándose entonces que el efecto causado por las lombrices en el medio fue efectivo llevando al aumento de los microorganismos mejorando la calidad del suelo tratado.

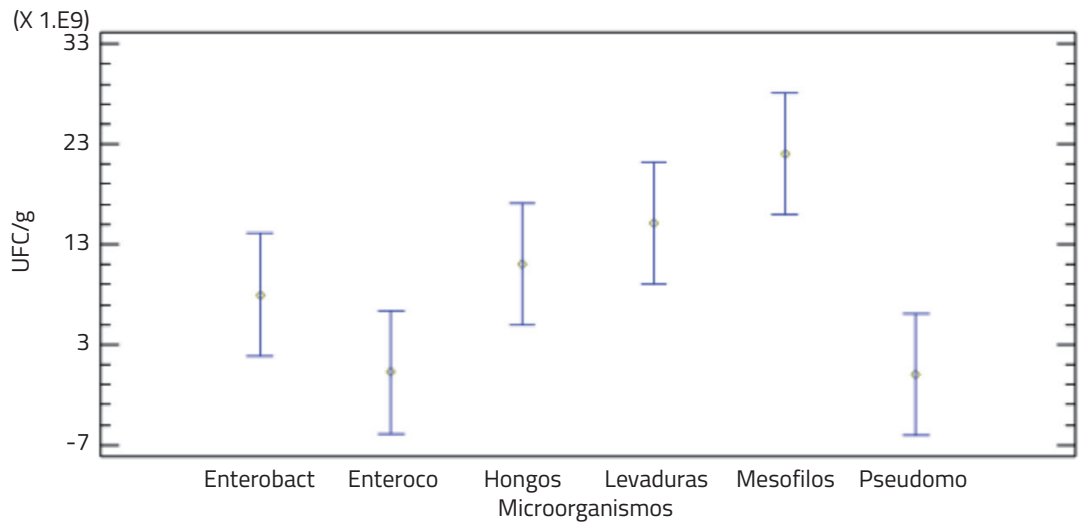

Figura 5. Gráfico de medias para las UFC por microorganismos evaluados.

Fuente: Elaboración propia.

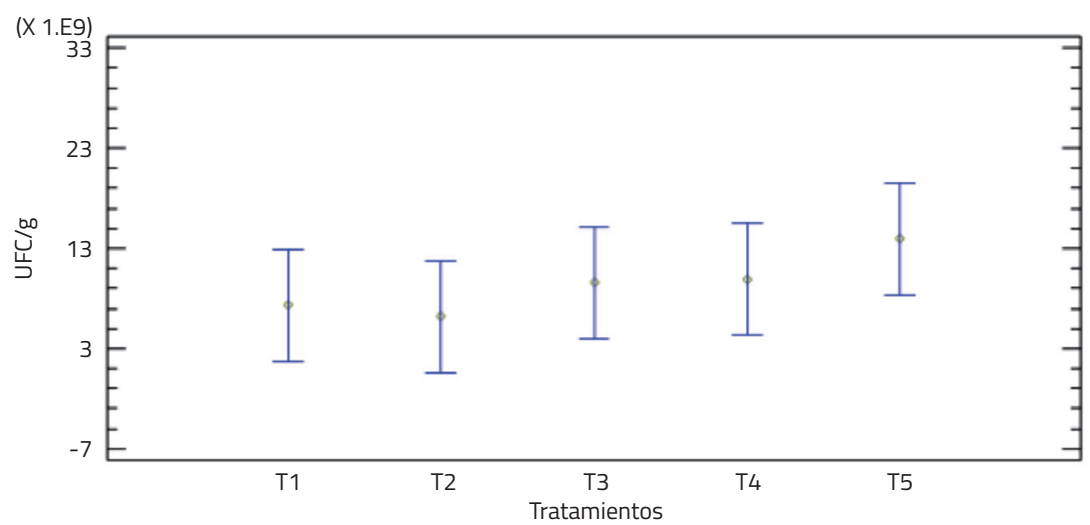

Figura 6. Gráfico de medias para las UFC por tratamientos evaluados.

Fuente: Elaboración propia. 


\section{CONCLUSIONES}

De acuerdo a los resultados, se puede observar un aumento de las unidades formadores de colonias en todos los microorganismos evaluados, mejorando las condiciones de calidad del suelo. Dicho comportamiento se hace evidente en todos los sistemas, a excepción del tratamiento sin lombrices, debido posiblemente a la muerte temprana de los microorganismos que estaban adaptados al medio en el momento de recolección de la muestra.

Deigual manera, se pudo observar que a medida quelaslombricesconsumíanlamateria orgánica disponible en el suelo se iba promoviendo el aumento de los microorganismos en todos los tratamientos evaluados, provocando esto una interacción de los mismos y mejorando las condiciones del suelo para que nuevas colonias de microorganismos se presentaran en los suelos tratados. Este comportamiento se debe principalmente a las deyecciones producidas por las lombrices que contribuyen a que se mejoren las condiciones químicas y físicas aportando nitrógeno, fósforo y potasio, con lo cual se mejora la fertilidad del suelo y se produce el aumento de la comunidad microbial del mismo, llevando a que al final del experimento se encontraran colonias en órdenes donde se considera que los suelos no presentan una contaminación.

\section{AGRADECIMIENTOS}

Los autores agradecen a la Institución Universitaria Colegio Mayor y a las personas que contribuyeron en la realización de este trabajo.

\section{REFERENCIAS}

[1] Brady, N. C. (2008). The natural and properties of soils. New York, USA: Macmillan Publishing Company.

[2] Kapusta, P., \& Sobczyk, Ł. (2015). Effects of heavy metal pollution from mining and smelting on enchytraeid communities under different land management and soil conditions. Sci. Total Environ., 536, pp. 517-526. doi: 10.1016/j.scitotenv.2015.07.086

[3] Luo, Y., Wu, L., Liu, L., Han, C., \& Li, Z. (2009). Heavy Metal Contamination and Remediation in Asian Agricultural Land, Natl. Inst. Agro-Environ. Sci. NIAES [Online]. Available: http://www.niaes.affrc.go.jp/marco/ marco2009/english/program/S-1 _ LuoYM.pdf. [Accessed: 10-Jun-2016].

[4] Singh, O. V., Labana, S., Pandey, G., Budhiraja, R., \& Jain, R. K. (2003). Phytoremediation: an overview of metallic ion decontamination from soil. Appl. Microbiol. Biotechnol., 61, pp. 405-412. doi: 10.1007/s00253-003-1244-4

[5] Ahmadpour, P., Ahmadpour, F., Mahmud, T. M. M., Abdu, A., Soleimani, M., \& Hosseini, F. (2012). Phytoremediation of heavy metals: A green technology. Afr. J. Biotechnol., 11, pp. 14036-14043. doi: 10.5897/AJB12.459

[6] Li, M. S., \& Yang, S. X. (2008). Heavy Metal Contamination in Soils and Phytoaccumulation in a Manganese Mine Wasteland, South China. Air Soil Water Res., 2008, pp. 31-41. doi: 10.4137/ASWR. S2041

[7] Clifton II, J. C. (2007). Mercury Exposure and Public Health. Pediatr. Clin. North Am., 54, pp. 237.e1-237.e45. doi: 10.1016/j. pcl.2007.02.005

[8] Spiegel, S. J., \& Veiga, M. M. (2010). International guidelines on mercury manage- 
ment in small-scale gold mining. J. Clean. Prod., 18, pp. 375-385. doi: 10.1016/j. jclepro.2009.10.020

[9] Leiva G., M. A., \& Morales, S. (2013). Environmental assessment of mercury pollution in urban tailings from gold mining. Ecotoxicol. Environ. Saf., 90, pp. 167-173. doi: 10.1016/j.ecoenv.2012.12.026

[10] Veiga, M. M., Maxson, P. A., \& Hylander, L. D. (2006). Origin and consumption of mercury in small-scale gold mining. J. Clean. Prod., 14, pp. 436-447. doi: 10.1016/j.jclepro.2004.08.010

[11] Carrasquero-Durán, A., \& Adams, M. (2003). Fraccionamiento de mercurio en suelos de áreas contaminadas de el callao, estado bolívar-venezuela. Agron. Trop., 53, pp. 331-346.

[12] Pinedo-Hernández, J., Marrugo-Negrete, J., \& Díez, S. (2015). Speciation and bioavailability of mercury in sediments impacted by gold mining in Colombia. Chemosphere, 119, pp. 1289-1295. doi: 10.1016/j.chemosphere.2014.09.044

[13] Shi, J., Liang, L., Jiang, G., \& Jin, X. (2005). The speciation and bioavailability of mercury in sediments of Haihe River, China. Environ. Int, 31, pp. 357-365. doi: 10.1016/j.envint.2004.08.008

[14] Wang, S., Jia, Y., Wang, S., Wang, X., Wang, H., Zhao, Z., \& Liu, B. (2009). Total mercury and monomethylmercury in water, sediments, and hydrophytes from the rivers, estuary, and bay along the Bohai Sea coast, northeastern China. Appl. Geochem., 24, pp. 1702-1711. doi: 10.1016/j. apgeochem.2009.04.037

[15] Hintelmann, H., Welbourn, P. M., \& Evans, R. D. (1995). Binding of methylmercury compounds by humic and fulvic acids. Water. Air. Soil Pollut., 80, pp. 1031-1034. doi: 10.1007/BF01189760

[16] Gochfeld, M. (2003). Cases of mercury exposure, bioavailability, and absorption. Ecotoxicol. Environ. Saf., 56, pp. 174-179. doi: 10.1016/S0147-6513(03)00060-5

[17] Knezović, Z., Trgo, M., \& Sutlović, D. (2016). Monitoring mercury environment pollution through bioaccumulation in meconium. Process Saf. Environ. Prot., 101, pp. 2-8. doi: 10.1016/j.psep.2016.01.013.

[18] Kurniati, E., Arfarita, N., Imai, T., Higuchi, T., Kanno, A., Yamamoto, K., \& Sekine, M. (2014). Potential bioremediation of mercury-contaminated substrate using filamentous fungi isolated from forest soil. J. Environ. Sci., 26, pp. 1223-1231. doi: 10.1016/S1001-0742(13)60592-6

[19] Croes, K., De Coster, S., De Galan, S., Morrens, B., Loots, I., Van de Mieroop, E., Nelen, V., Sioen, I., Bruckers, L., Nawrot, T., Colles, A., Hond, E. D., Schoeters, G., van Larebeke, N., Baeyens, W., \& Gao, Y. (2014). Health effects in the Flemish population in relation to low levels of mercury exposure: From organ to transcriptome level. Int. J. Hyg. Environ. Health, 217, pp. 239-247. doi: 10.1016/j. ijheh.2013.06.004

[20] Nakazawa, K., Nagafuchi, O., Kawakami, T., Inoue, T., Yokota, K., Serikawa, Y., Cyio, B., \& Elvince, R. (2016). Human health risk assessment of mercury vapor around artisanal small-scale gold mining area, Palu city, Central Sulawesi, Indonesia. Ecotoxicol. Environ. Saf., 124, pp. 155-162. doi: 10.1016/j.ecoenv.2015.09.042

[21] Chan, M., \& Grace, M. E. (2004) Fish Consumption, Mercury Exposure, and Heart Diseases. Nutr. Rev., 62, pp. 68-72. doi: 10.1111/j.1753-4887.2004.tb00027.x

[22] Gundacker, C., Wittmann, K. J., Kukuckova, M., Komarnicki, G., Hikkel, I., \& Gencik, M. (2009). Genetic background of lead and mercury metabolism in a group of medical students in Austria. Environ. 
Res., 109, pp. 786-796. doi: 10.1016/j. envres.2009.05.003

[23] Stern, A. H. (2005). A review of the studies of the cardiovascular health effects of methylmercury with consideration of their suitability for risk assessment. Environ. Res., 98, pp. 133-142. doi: 10.1016/j. envres.2004.07.016

[24] Kensa, V. M. (2011). Bioremediation - An overview. J. Ind. Pollut. Controll, 27, pp. 161-168.

[25] Lemtiri, A., Colinet, G., Alabi, T., Cluzeau, D., Zirbes, L., Haubruge, E., \& Francis, F. (2014). Impacts of earthworms on soil components and dynamics. A review. Biotechnol Agron Soc Env., 18, pp. 121-133.

[26] Lemtiri, A., Liénard, A., Alabi, T., Brostaux, Y., Cluzeau, D., Francis, F., \& Colinet, G. (2016). Earthworms Eisenia fetida affect the uptake of heavy metals by plants Vicia faba and Zea mays in metal-contaminated soils. Appl. Soil Ecol., 104, pp. 67-78. doi: 10.1016/j.apsoil.2015.11.021

[27] Vera, J. A. (2013). Bioremediation treatments for disposal of waste generated of inorganic salts general chemistry laboratory using technical vermiculture. Rev. Ambient. Agua, Aire Suelo, 4, pp. 33-41.

[28] Tuomela, M., Lyytikäinen, M., Oivanen, P., \& Hatakka, A. (1998). Mineralization and conversion of pentachlorophenol (PCP) in soil inoculated with the white-rot fungus Trametes versicolor. Soil Biol. Biochem., 31, pp. 65-74. doi: 10.1016/S00380717(98)00106-0

[29] Lin, Z., Zhen, Z., Wu, Z., Yang, J., Zhong, L., Hu, H., Luo, C., Bai, J., Li, Y., \& Zhang, D. (2016). The impact on the soil microbial community and enzyme activity of two earthworm species during the bioremediation of pentachlorophenol-contaminated soils. J. Hazard. Mater., 301, pp. 35-45. doi: 10.1016/j.jhazmat.2015.08.034
[30] Zhang, B.-G., Li, G.-T., Shen, T.-S., Wang, J.-K., \& Sun, Z. (2000). Changes in microbial biomass $C, N$, and $P$ and enzyme activities in soil incubated with the earthworms Metaphire guillelmi or Eisenia fetida. Soil Biol. Biochem., 32, pp. 2055-2062. doi: 10.1016/S0038-0717(00)00111-5

[31] Li, X., Lin, Z., Luo, C., Bai, J., Sun, Y., \& Li, Y. (2015). Enhanced microbial degradation of pentachlorophenol from soil in the presence of earthworms: Evidence of functional bacteria using DNA-stable isotope probing. Soil Biol. Biochem., 81, pp. 168-177. doi: 10.1016/j.soilbio.2014.11.011

[32] Suthar, S. (2007). Nutrient changes and biodynamics of epigeic earthworm Perionyx excavatus (Perrier) during recycling of some agriculture wastes. Bioresour. Technol., 98, pp. 1608-1614. doi: 10.1016/j. biortech.2006.06.001

[33] Rodger, B. B. (2012). Standard Methods for the Examination of Water and Wastewater, Washington D. C, USA. Joint Editorial Board.

[34] Society for General Microbiology. (2006). Basic Practical Microbiology, Reading, England. Society for General Microbiology.

[35] Almanya, S. (2010). Transformación de sólidos provenientes de lodos generados en el sistema de alcantarillado de Bogotá mediado lombriz roja californiana (Esenia foetida). Universidad del Bosque.

[36] Escobar, A. (2013). Usos potenciales del humus (abono orgánico lixiviado y sólido) en la empresa fertilombriz. Caldas, Colombia. Corporación Universitaria Lasallista.

[37] Díaz, L. P., Medina, L. F., Latife, J., Digonzelli, P. A., \& Sosa, S. B. (2004). Aclimatación de plantas micropropagadas de caña de azúcar utilizando el humus de lombriz. Ria, 32, pp. 115-128. 
[38] Medina, L. F., Jaime, M., Chueca, C., Bocanera, B., Toro, F., \& Mascaró, P. (2001). "Presencia y cuantificación de Azotobacter sp. y Azospirillum sp. en lombricompuesto". Presentado en Segunda Reunión de Producción Vegetal del NOA, San Miguel de Tucumán, Argentina.

[39] Mahbub, K. R., Krishnan, K., Megharaj, M., \& Naidu, R. (2016). Bioremediation potential of a highly mercury resistant bacterial strain Sphingobium SA2 isolated from contaminated soil. Chemosphere, 144, pp. 330-337. doi: 10.1016/j. chemosphere.2015.08.061

[40] Soto, C., Gutiérrez, S., Rey-León, A., \& González-Rojas, E. (2010). Biotransformación de metales pesados presentes en lodos ribereños de los ríos Bogotá y Tunjuelo. NOVA - Publ. Científica En Cienc. Bioméd., 8, pp. 195-205.

[41] Hinton, J. J., \& Veiga, M. M. (2009). Using earthworms to assess hg distribution and bioavailability in gold mining soils. Soil and Sediment Contamination: An International Journal, 18, pp. 512-524. 